PROCEEDINGS OF THE AMERICAN MATHEMATICAL SOCIETY

Volume 124, Number 5, May 1996

\title{
CONGRUENCE LATTICES OF ALGEBRAS- THE SIGNED LABELLING
}

\author{
S. W. SEIF \\ (Communicated by Lance W. Small)
}

\begin{abstract}
For an arbitrary algebra $\mathbf{A}$ a new labelling, called the signed labelling, of the Hasse diagram of Con $\mathbf{A}$ is described. Under the signed labelling, each edge of the Hasse diagram of Con $\mathbf{A}$ receives a label from the set $\{+,-\}$. The signed labelling depends completely on a subset of the unary polynomials of $\mathbf{A}$ and its inspiration comes from semigroup theory. For finite algebras, the signed labelling complements the labelled congruence lattices of tame congruence theory (TCT). It provides a different kind of information about those algebras than the TCT labelling particularly with regard to congruence semimodularity. The main result of this paper shows that the congruence lattice of any algebra $\mathbf{A}$ admits a natural join congruence, denoted $\approx_{+}$, such that Con $\mathbf{A} / \approx_{+}$satisfies the semimodular law. In an application of that result, it is shown that for a regular semigroup $\mathbf{S}$, for which $\mathcal{J}=\mathcal{D}$ in $\mathbf{H}(\mathbf{S}), \approx_{+}$is actually a lattice congruence, $\approx_{+}$coincides with $U$, and $\operatorname{Con} \mathbf{S} / U\left(=\operatorname{Con} \mathbf{S} / \approx_{+}\right)$ satisfies the semimodular law.
\end{abstract}

\section{INTRODUCTION}

Classical algebras (groups, rings, etc.) rely for their definitions on a limited number of binary operations. These operations satisfy certain identities extrapolated (more or less) from number systems. A more general view of an algebra is this: An algebra $\mathbf{A}$ is a set $\mathbf{A}$ equipped with a set of fundamental operations, $\left\{f_{i}: i \in I\right\}$, where $I$ is an index set. Each $f_{i}(i \in I)$ is just a function $f_{i}: A^{n_{i}} \rightarrow A$, where $n_{i} \in \mathbb{N}$. Congruences and congruence lattices (defined below) of algebras provide a systematic means of uncovering structure in an algebra or a class of algebras. In the early 80 's the congruence lattice invariant was significantly sharpened for finite algebras by D. Hobby and R. McKenzie $[\mathrm{H}-\mathrm{M}]$ via what they called labelled congruence lattices. A labelling is a mapping from covering pairs of congruences to a set of labels. For a covering pair, its associated label can be affixed to the edge between the pair in the Hasse diagram of the congruence lattice. In [H-M] the authors produced the aforementioned labelling with labels $\{1,2,3,4,5\}$ as part of their tame congruence theory. The labelling we describe here, called the signed labelling, is considerably easier to effect than that of $[\mathrm{H}-\mathrm{M}]$ since it depends only on a subset of the unary polynomials while the other seems to reflect the deepest structural influences of the binary polynomials. To each covering pair in the congruence lattice of a finite algebra, the $[\mathrm{H}-\mathrm{M}]$ labelling associates one of the following

Received by the editors September 21, 1993 and, in revised form, September 7, 1994.

1991 Mathematics Subject Classification. Primary 08A30.

Key words and phrases. Congruence lattice, semimodularity. 
classes of well-studied algebras: Abelian groups (type 2), Boolean algebras (type 3), distributive lattices (type 4), semilattices (type 5), and "essentially unary" (type 1). The $[\mathrm{H}-\mathrm{M}]$ labelling indicates that even a random algebra - select a finite set and some functions on it - can admit interpretation from an "algebraic" point of view. Our analysis of algebra is from a different point of view and treats algebras as pre-ordered objects, generalizing the well-studied pre-order associated with the $\mathcal{J}$ relation on a semigroup to arbitrary algebras. Associated with the pre-order here are two equivalence relations (the so-called $\mathcal{J}$ and $\mathcal{K}$ relations). The signed labelling measures the influence that the pre-order and the $\mathcal{J}$ and $\mathcal{K}$ relations exert on the algebra, particularly its congruences and congruence lattice. The signed labelling has antecedents. In $[\mathrm{A}-\mathrm{K}]$, starting with a variety $V$, the authors put to use a generalization of the $\mathcal{J}$ relation to the algebras of $V$. From $[R]$, we have generalized results that lead to a main tool here (Proposition 1.11). From [J1] and [J2] came an interest in congruence semimodularity.

We offer a number of applications of the signed labelling; each involves the role of semimodularity in congruence lattices. A certain join congruence $\approx_{+}$is described here (through the signed labelling) and it turns out for an arbitrary algebra $\mathbf{A}$, Con $\mathbf{A} / \approx_{+}$satisfies the semimodular law (as a join semilattice). We are able to give a representation via signed congruence lattices of an important congruence on congruence lattices of a regular semigroup $\mathbf{S}$ (the $U$ congruence) under the proviso that all homomorphic images of $\mathbf{S}$ satisfy $\mathcal{J}=\mathcal{D}$. We prove here a new result: If $\mathbf{S}$ is regular and all homomorphic images of $\mathbf{S}$ satisfy $\mathcal{J}=\mathcal{D}$, then Con $\mathbf{S} / U$ satisfies the semimodular law.

In a sequel to this work [S], using signed congruence lattices, we give characterizations of algebras whose congruence lattices satisfy the semimodular law and those whose congruence lattices satisfy the Jordan Chain condition.

\section{The SIGNED LABELLING}

An algebra $\mathbf{A}=\left\langle A, f_{1}, \ldots, f_{i}, \ldots\right\rangle$ is a set $A$ equipped with a set of fundamental operations $f_{1}, \ldots, f_{i}, \ldots\left(f_{i}: A^{n_{i}} \rightarrow A, n_{i} \in \mathbb{N}, i=1, \ldots\right.$, where $n_{i}$ is said to be the arity of $f_{i}$ ). One way to analyze the structure of $\mathbf{A}$ is through its congruence lattice. An equivalence relation $\theta$ on $A$ is a congruence if for every fundamental operation $f_{i}$, if $\left(a_{j}, b_{j}\right) \in \theta, j=1, \ldots, n_{i}$, then $\left(f_{i}\left(a_{1}, \ldots, a_{n_{i}}\right), f_{i}\left(b_{1}, \ldots, b_{n_{i}}\right)\right) \in \theta$. The congruences of $\mathbf{A}$, Con $\mathbf{A}$, are partially ordered under inclusion and constitute a sublattice of $E q A$, the lattice of equivalence relations on $A$. To each congruence $\theta$ is associated the quotient algebra $\mathbf{A} / \theta$. The underlying set of $\mathbf{A} / \theta$ is the set $A / \theta$ of all equivalence classes of $\theta$. We have the mapping $h_{\theta}: A \rightarrow A / \theta$ which maps each $a \in A$ to the equivalence class $a / \theta$ containing $a$. The operations $\bar{h}_{1}, \ldots, \bar{h}_{i}, \ldots$ of $A / \theta$ are defined in the only way possible so that $h_{\theta}$ is a homomorphism of $\mathbf{A}$ onto $\mathbf{A} / \theta$. For $a \in A$, let $\bar{a} / \theta$ and denote by $h_{\theta}$ the map from $A$ onto its congruence classes.

The diagonal relation is written $\Delta$; the universal relation is denoted $\nabla$. Both are of course congruences. For congruences $\alpha$ and $\beta$, if $\beta$ covers $\alpha$ in Con $\mathbf{A}$, write $\beta \succ \alpha$. For congruences $\gamma>\mu,[\mu, \gamma]=\{\theta \in \operatorname{Con} \mathbf{A}: \gamma \geq \theta \geq \mu\}$. For $a, b \in A$, the (unique) minimal congruence identifying $a$ and $b$ is denoted $\theta(a, b)$. If $\gamma>\mu$ in Con $\mathbf{A}$, the congruence on $\mathbf{B} / \mu$ induced by $\gamma$ is denoted $B / \mu$.

We describe below the signed congruence lattice of $\mathbf{A}$. To each edge of the Hasse diagram (i.e., each covering pair of Con $\mathbf{A}$ ) we affix + or - . The assignment 
is natural and not difficult once several elementary lemmas and definitions are in place.

Definition 1.1. For an algebra $\mathbf{A}=\left\langle A, f_{1}, \ldots, f_{i}, \ldots\right\rangle$ a certain monoid of transformations on $A$ is defined. For each fundamental operation $f_{i}$ with arity $n_{i}>0$ is defined a set of unary functions on $A: F_{i}$ is the set of all functions $x \rightarrow f_{i}$ $\left(a_{1}, \ldots, a_{k-1}, x, a_{k+1}, \ldots, a_{n_{i}}\right)$ where $k \in\left\{1, \ldots, n_{i}\right\}$ and $a_{1}, \ldots, a_{n_{i}} \in A$. We let $M(\mathbf{A})$ be the monoid of self-maps of $A$ generated by the union of these sets of functions.

Example 1.2. Suppose $\mathbf{A}$ is a semigroup. It is easy to verify that $M(\mathbf{A})$ is the set of all functions $x \rightarrow a x b$ with $a, b \in A^{1}=A \cup\{1\}$. For a more general binary algebra, $M(\mathbf{A})$ can be greatly more complicated.

Two important observations concerning $M(\mathbf{A})$ follow. Firstly, consider the algebra $\mathbf{A}^{*}=\langle A, M(\mathbf{A})\rangle$. It is not difficult to verify that an equivalence relation $\theta$ on $A$ is a congruence on $\mathbf{A}$ if and only if $\theta$ is a congruence on $\mathbf{A}^{*}$. Secondly, suppose $\theta$ is a congruence on $\mathbf{A}$. Consider the algebra $\mathbf{B}=\mathbf{A} / \theta$. Observe that the map from $M(\mathbf{A})$ to $M(\mathbf{B})$ given by $f \mapsto \bar{f}$ is a monoid homomorphism, where $\bar{f}$ is the function induced by $f$ on $B=A / \theta$. Notice that the well-definedness of $\bar{f}$ follows from the fact that $\theta$ is a congruence of $\mathbf{A}^{*}$.

We define a pre-order on $\mathbf{A}$ determined by $M(\mathbf{A})$ and which induces two equivalence relations on $A$. The object of these definitions is to place a "frame" on an arbitrary algebra.

Definition 1.3. (i) For $a, b \in A$, we say $a \geq b$ if there exists $f \in M(\mathbf{A})$ such that $f(a)=b$.

(ii) If $a \geq b$ and $b \geq a$, we say that $a$ and $b$ are $\mathcal{J}$ related and write $(a, b) \in \mathcal{J}$.

(iii) Let $(a, b) \in \mathcal{J}$. If for all $f \in M(\mathbf{A}),(f(a), a) \in \mathcal{J}$ if and only if $(f(b), b) \in \mathcal{J}$, then we say that $a$ and $b$ are $\mathcal{K}$ related and we write $(a, b) \in \mathcal{K}$.

It is easy to verify that $\geq$ is a pre-order, $\mathcal{J}$ and $\mathcal{K}$ are equivalence relations, and $\mathcal{K} \leq \mathcal{J}$. Observe that the $\mathcal{J}$ classes are partially ordered. The following will be used frequently. Suppose $(a, b) \in \mathcal{J}-\mathcal{K}$ and $f \in M(\mathbf{A})$ is such that $(a, f(a)) \in \mathcal{J}$, $(b, f(b)) \notin \mathcal{J}$ (so $b>f(b))$. Since $(a, f(a)) \in \mathcal{J}$, there exists $g \in M(\mathbf{A})$ such that $g f(a)=a$ (and $b>g f(b)$ ). Thus we see that $(a, b) \in \mathcal{J}-\mathcal{K}$ if and only if $(a, b) \in \mathcal{J}$, and there exists $h \in M(\mathbf{A})$ such that either $h(a)=a, b>h(b)$ or $h(b)=b, a>h(a)$.

Example 1.4. (i) We describe $\mathcal{J}, \mathcal{K}$ on a five-element unary algebra $\mathbf{C}$. Let $C=$ $\{a, b, c, d, 0\}$, and consider functions $f, g \in C^{C}$ with $f(a)=b, f(b)=a, f(c)=d$, $f(d)=c, f(0)=0 ; g(a)=c, g(c)=c, g(b)=g(d)=g(0)=0$. The decomposition of $B$ into $\mathcal{J}$ classes is given by $\{0\}<\{c, d\}<\{a, b\}$. Notice that $(a, b) \in \mathcal{K}$ but $(c, d) \in \mathcal{J}-\mathcal{K}, g$ witnessing that $(c, d) \notin \mathcal{K}$.

(ii) If $\mathbf{A}$ is a semigroup, then it is easy to verify that the $\mathcal{J}$ relation from semigroup theory ( $a \mathcal{J} b$ if $A^{1} a A^{1}=A^{1} b A^{1}$ ) and the $\mathcal{J}$ relation defined here are the same.

For $a \in A$, let $J_{a}$ denote the $\mathcal{J}$-class of $a$. For a congruence $\theta$ on $\mathbf{A}, \mathcal{J}^{\theta}$ denotes the $\mathcal{J}$ relation on $\mathbf{A} / \theta$; for $\bar{a} \in \mathbf{A} / \theta, J_{\bar{a}}$ is the $\mathcal{J}^{\theta}$ class of $\bar{a}$; and put $J^{\bar{a}}=h_{\theta}^{-1}\left(J_{\bar{a}}\right)$. The same conventions are followed for $\mathcal{K}$. Lemma 1.5 below describes for $\theta \in$ Con $\mathbf{A}$ how $\mathcal{J}$ and $\mathcal{J}^{\theta}$ are related. See [R] for results on $\mathcal{J}$ classes of semigroups of the type in Lemma 1.5 below. Lemma 1.5(iii) will not be used here. It is of importance 
especially when A satisfies the Descending Chain condition on its $\mathcal{J}$ classes and is used in [S].

Lemma 1.5. Let $\mathbf{A}$ be an algebra, $\theta$ a congruence of $\mathbf{A}$ and $\mathbf{B}=\mathbf{A} / \theta$.

(i) $a \geq b$ implies $\bar{a} \geq \bar{b}$. In particular, $(a, b) \in \mathcal{J}$ implies $(\bar{a}, \bar{b}) \in \mathcal{J}^{\theta}$.

(ii) $J^{\bar{a}}$ is a union of $\mathcal{J}$ classes (of $A$ ).

(iii) Let $J^{\bar{a}}=\bigcup_{i \in I} J_{i}$, where $I$ is an index set and for all $i \in I, J_{i}$ is a $\mathcal{J}$ class. Suppose $J$ is minimal in $\left\{J_{i}: i \in I\right\}$.

Then for any $\bar{u} \in J_{\bar{a}}$, there exists $v \in J$ such that $\bar{v}=\bar{u}$. In particular, $J / \theta$ is in one-to-one correspondence with $J_{\bar{a}}$. Moreover, for a $\mathcal{K}$ class $K$, with $K$ contained in $J$, if $\{c, d\} \subseteq J$, then $(c, d) \in \mathcal{K}$ if and only if $(\bar{c}, \bar{d}) \in \mathcal{K}^{\theta}$.

Proof. (i) If $a \geq b$ in $\mathbf{A}$, then there exists $f \in M(\mathbf{A})$ such that $f(a)=b$. Consider $\bar{f} \in M(\mathbf{B})$. Since $\bar{f}(\bar{a})=\bar{b}, \bar{a} \geq \bar{b}$ in $\mathbf{B}$.

(ii) Suppose $c \in J^{\bar{a}}$ and $(c, d) \in \mathcal{J}$. It suffices to show that $d \in J^{\bar{a}}$. By hypothesis $(\bar{a}, \bar{c}) \in \mathcal{J}^{\theta}$ and by (i) above $(c, d) \in \mathcal{J}$ implies $(\bar{c}, \bar{d}) \in \mathcal{J}^{\theta}$. Since $\mathcal{J}^{\theta}$ is an equivalence relation, $(\bar{a}, \bar{d}) \in \mathcal{J}^{\theta}$ and $d \in J^{\bar{a}}$ by definition.

(iii) Let $J$ be a minimal $\mathcal{J}$-class in $J^{\bar{a}}$ and $\bar{u} \in J_{\bar{a}}$. Choose any $c \in J$. Since $J \subseteq J^{\bar{a}},(\bar{c}, \bar{u}) \in \mathcal{J}^{\theta}$. Now $(\bar{c}, \bar{u}) \in \mathcal{J}^{\theta}$ implies that there exists $\bar{f} \in M(\mathbf{B})$ such that $\bar{f}(\bar{c})=\bar{u}$. Letting $v=f(c)$ in $\mathbf{A}$, we have $c, v \in J^{\bar{a}}$ and $c \geq f(c)=v$. By minimality of $J, v \in J$. The last sentence in (iii) remains to be proved. Suppose $\{c, d\} \subseteq J, f \in M(\mathbf{A})$. Then by minimality of $J,(f(c), c) \in \mathcal{J}$ if and only if $(\bar{f}(\bar{c}), \bar{c}) \in \mathcal{J}^{\theta}$ from which the equivalence in that sentence follows.

Example 1.6. The lemma above indicates that $\mathcal{J}$ is well behaved under homomorphism; however, this is less the case for $\mathcal{K}$. Consider $\mathbf{C}$ in Example 1.4 above with partition $a c|b d| \theta$. It is easy to verify that $\theta$ is a congruence of $\mathbf{C}$ (see Figure 1). The $\mathcal{J}^{\theta}$ decomposition is $\{\bar{\theta}\}<\{\bar{a}, \bar{b}\}$. Note that $\bar{g}(\bar{a})=\bar{a}$ but $\bar{g}(\bar{b})=\overline{0}$. In particular, $(a, b) \in \mathcal{K}$ but $(\bar{a}, \bar{b}) \notin \mathcal{K}^{\theta}$. Also, in $\mathbf{C}, K^{\bar{a}}=\{a, c\}$ which is not a union of $\mathcal{K}$ classes of $\mathbf{C}$. So both 1.5(i) and (ii) fail with $\mathcal{K}$ in the place of $\mathcal{J}$.

For a subset $S$ of $A$, let $S^{c}$ denote its complement in $A$.

Definition 1.7. Let $\theta$ be an equivalence relation.

(i) The content of $\theta$, denoted $C(\theta)$, is $\{(u, v) \in \theta: u \neq v\}$.

(ii) $J(\theta)=\{J \in A / \mathcal{J}:(J \times A) \cap C(\theta) \neq \theta\}$.

(iii) $J^{*}(\theta)=\left\{J \in A / \mathcal{J}:\left(J \times J^{c}\right) \cap C(\theta) \neq \theta\right\}$.

For example, with $\mathbf{C}$ and $\theta$ as in Example 1.6,

$$
C(\theta)=\{(a, c),(c, a)(b, d),(d, b)\}
$$

and $J(\theta)=\left\{J_{a}, J_{c}\right\}=J^{*}(\theta)$. For $\beta$ in Figure $1, J^{*}(\beta)$ is properly contained in $J(\beta)$. Next, a congruence generation lemma, whose proof is elementary, is stated. For a subset $R$ of $A \times A$, let $\{R\}^{*}$ denote the symmetric, transitive, reflexive closure of $R$.

Lemma 1.8. For $a, b \in A, \theta(a, b)=\{(f(a), f(b)): f \in M(A)\}^{*}$.

Definition 1.9. (i) Let $I$ be a subset of $A$. Then $I$ is said to be an ideal of $A$ if $v \in I, v \geq w$ imply $w \in I$.

(ii) Let $a \in A$. The principal ideal generated by $a$, denoted by $a]$, is $\{u \in A: a \geq$ $u\}$. Also, let $a)=\{u \in A: a>u\}$. 
Filters, principal filters, etc. can be dually defined. Note that the complement of ideals are filters and conversely; that both filters and ideals are unions of $\mathcal{J}$-classes; and that the set of ideals is closed under union and intersection. To an ideal $I$ we may associate a congruence $\theta_{I}=\{(u, v) \in A \times A:\{u, v\} \subset I$ or $u=v\}$.

Lemma 1.10. Let $a, b, c, d \in A$ with $a \neq b$.

(i) $(a, b) \in \theta(c, d)$ implies $a] \cup b] \subseteq c] \cup d]$ (that is, $\{a, b\} \subseteq c] \cup d]$ ).

(ii) Suppose $(a, b) \in \mathcal{K}$ and let $J=J_{a}$. Then $\left.\left.\left.C(\theta(a, b)) \subseteq \mathcal{K}\right|_{J} \cup(a) \times a\right)\right)$.

(iii) Let $K$ be a $\mathcal{K}$-class. Then $K$ is a congruence class of some congruence.

Proof. For (i), since $a \neq b$, by Lemma 1.8 there must exist $f, g \in M(\mathbf{A})$ such that $a \in\{f(c), f(d)\}$ and $b \in\{g(c), g(d)\}$; (ii) and (iii) follow from Lemma 1.8 and the definition of $\mathcal{K}$. In fact, $K$ is a congruence class of the least congruence identifying all pairs in $K$.

A congruence $\theta$ is said to be minimal if $\theta \succ \Delta$. The first small surprise here is that the $\mathcal{J}$ structure of $\mathbf{A}$ imposes strong restrictions on minimal congruences of A. The idea for the following proposition and its proof come from results in finite semigroup theory $[R]$ (which were later extended to arbitrary semigroups in $[\mathrm{D}-\mathrm{K}]$ ). Note that if $\theta$ is minimal and $(a, b) \in C(\theta)$, then $\theta=\theta(a, b)$.

Proposition 1.11. Let $\theta$ be a minimal congruence. Then $\theta$ satisfies exactly one of:

(i) $\theta \leq \mathcal{K}, J(\theta)=\{J\}$ for some $\mathcal{J}$ class $J$ (see Definition 1.7).

(ii) $\theta \cap \mathcal{K}=\Delta, J(\theta)=\left\{J_{1}, J_{2}\right\}$, and $J_{1} \neq J_{2}$. If $J_{1} \ngtr J_{2}$, then $C(\theta) \cap\left(J_{1} \times J_{1}\right)=$ $\varnothing$. Moreover, if $J_{2}>J_{1}$, then $J_{2}$ covers $J_{1}\left(J_{2} \succ J_{1}\right)$ in the partial order defined on the $\mathcal{J}$ classes.

Proof. First suppose there exists $(a, b) \in(\theta \cap \mathcal{K})-\Delta$. Since $\theta$ is minimal, $\theta=\theta(a, b)$. For any $f \in M(\mathbf{A}),(a, b) \in \mathcal{K}$ implies $\{f(a), f(b)\} \subseteq J_{a}$ or $\{f(a), f(b)\} \cap J_{a}=\varnothing$. Suppose $\{f(a), f(b)\} \cap J_{a}=\varnothing$. Then $a>f(a)$ and $a>f(b)$, with a similar statement for $b$ also holding. Hence $a] \cup b] \nsubseteq f(a)] \cup f(b)]$. By Lemma 1.10(i), $\theta(a, b)>\theta(f(a), f(b))$. Since $\theta(a, b)=\theta$ is minimal, $\theta(f(a), f(b))=\Delta$; hence $f(a)=f(b)$. That is, for any $f \in M(A),\{f(a), f(b)\} \nsubseteq J_{a}$ implies $f(a)=f(b)$ which in turn implies that $J(\theta)=\left\{J_{a}\right\}$. By Lemma 1.10(ii) it also follows that $\theta \leq \mathcal{K}$.

Now assume that no pair in $C(\theta)$ is in $\mathcal{K}$. Let $(a, b) \in C(\theta)$. If $(a, b) \in \mathcal{J}$, since $(a, b) \notin \mathcal{K}$, we may assume there exists $f \in M(A)$ such that $f(a) \in J_{a}$ but $f(b) \notin J_{b}$. In that case, $(f(a), f(b)) \in C(\theta)$ and $(f(a), f(b)) \notin \mathcal{J}$. This shows that $|J(\theta)| \geq 2$. So we may as well assume $(a, b) \in C(\theta)-\mathcal{J}$. Let $(c, d) \in C(\theta)$. Then $\theta(c, d)=\theta=\theta(a, b)$, so by Lemma 1.10(i) $a] \cup b]=c] \cup d]$ from which it follows readily that $\left\{J_{a}, J_{b}\right\} \supseteq\left\{J_{c}, J_{d}\right\}$. We have shown that $|J(\theta)|=2$. Now suppose $J(\theta)=\left\{J_{a}, J_{b}\right\}, J_{a} \ngtr J_{b},(s, t) \in\left(J_{a} \times J_{a}\right) \cap \theta$. We show $s=t$. Again, we have $s] \cup t] \subseteq a] \cup b]$ with equality holding if $\theta(s, t)=\theta(a, b)$. Since $b \notin s] \cup t]$, it follows that $\theta(s, t) \neq \theta(a, b)$; hence $s=t$. Lastly, suppose $J_{b}>J_{a}$. We show next that $J_{b} \succ J_{a}$. Let $c$ be such that $J_{b}>J_{c} \geq J_{a}$. Then $b>c$ implies that there exists $f \in M(A)$ such that $f(b)=c$. Notice that $b \notin c=f(a)] \cup a]$. By Lemma 1.10(i), applied to $(f(a), f(b))=(f(a), c)$, we have $c=f(b)=f(a)$, so $J_{a} \geq J_{c}$; hence, $J_{b} \succ J_{a}$.

The signed labelling of the Hasse diagram of a congruence lattice can now be defined. 
Definition 1.12. (i) For $\beta \succ \alpha$ in $\operatorname{Con} \mathbf{A}$, we say that $t\langle\alpha, \beta\rangle=+$ if $\beta / \alpha \leq \mathcal{K}^{\alpha}$. Otherwise $t\langle\alpha, \beta\rangle=-$.

(ii) Let $[\mu, \gamma]$ be an interval in Con A. Then $t\{\mu, \gamma\}=\left\{t\left\langle\mu_{0}, \gamma_{0}\right\rangle: \mu \leq \mu_{0} \prec \gamma_{0} \leq\right.$ $\gamma\}$.

(iii) Let $t\{\Delta, \nabla\}=t\{\mathbf{A}\}$.

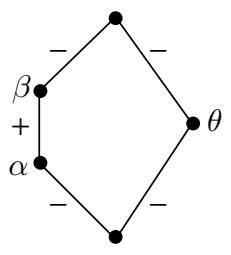

FigurE 1. The labelled congruence lattice of $\mathbf{C}$ (of Example 1.4): $\alpha=0 c d / a / b ; \beta=0 c d / a b ; \theta=a c / b d / 0$.

Note that by its definition the typing of $\mathbf{B}=\mathbf{A} / \alpha$ coincides with that of the typing of the filter $[\alpha, \nabla]$ in Con $\mathbf{A}$. The next proposition is crucial. It says that a certain global property holds if and only if it holds at the local level.

Proposition 1.13. Let $\mathbf{A}$ be any algebra and $\gamma>\mu$ in Con $\mathbf{A}$. Let $\mathbf{B}=\mathbf{A} / \mu$. Then the following are equivalent.

(i) $\gamma / \mu \leq \mathcal{K}^{\mu}$.

(ii) $\gamma / \mu \leq \mathcal{J}^{\mu}$.

(iii) $t\{\mu, \gamma\}=\{+\}$.

Proof. Notice that (i) $\Rightarrow$ (ii) is immediate since $\mathcal{K}^{\mu} \leq \mathcal{J}^{\mu}$. For (ii) $\Rightarrow$ (iii) we have that if $\gamma / \mu \leq \mathcal{J}^{\mu}$ and $\gamma \leq \mu_{0} \prec \gamma_{0} \leq \mu$, then $\gamma_{0} / \mu_{0} \leq \mathcal{J}^{\mu_{0}}$ by Lemma 1.5(i). By Proposition 1.11, $\gamma_{0} / \mu_{0} \leq \mathcal{J}^{\mu_{0}}$ implies $\gamma_{0} / \mu_{0} \leq \mathcal{K}^{\mu_{0}}$, so by definition $t\left\langle\mu_{0}, \gamma_{0}\right\rangle=+$. This proves (ii) $\Rightarrow$ (iii).

For (iii) $\Rightarrow(\mathrm{i})$, notice first that we may assume $\mu=\Delta$. So suppose $t\{\Delta, \gamma\}=\{+\}$ and for contradiction assume $\gamma \not \leq \mathcal{K}$. Since $\gamma \not \leq \mathcal{K}$, it follows that $\gamma \not \leq \mathcal{J}$. So there exists $(a, b) \in \gamma-\mathcal{J}$. We assume without loss of generality that $b \not a$. Let $\beta$ be the congruence generated by $\{(u, v) \in \theta(a, b):\{u, v\} \subseteq a) \cup b]$ or $\left.\left.\{u, v\} \in \mathcal{K}\right|_{J_{a}}\right\}$. In particular, by Lemma 1.10(ii), $\left.\left.\left.\left.C(\beta) \subseteq\left(J_{a} \times J_{a}\right) \cup(a) \times b\right]\right) \cup(b] \times a\right)\right)$ from which it follows that $(a, b) \notin \beta$. In fact we claim that $\theta(a, b)$ covers $\beta$. To see this, let $(u, v) \in \theta(a, b)-\beta$. So $\{u, v\} \cap J_{a} \neq \varnothing$; moreover, $(u, v) \notin \mathcal{K}$ (otherwise $\left.(u, v) \in \beta\right)$. Since $(u, v) \notin \mathcal{K}$, it follows that there exists $w \in A$ such that $(a, w) \in \theta(u, v)$ and $a>w$ or $w \leq b$. By the definition of $\beta,(b, w) \in \beta$; hence, $(a, b) \in \beta \vee \theta(u, v)$ and so $\theta(a, b)=\beta \vee \theta(u, v)$. It follows that $\theta(a, b)$ covers $\beta$. From the definition of $\beta$, it is easy to see that $(\bar{a}, \bar{b}) \notin \mathcal{J}^{\beta}$ and so $t\langle\beta, \theta(a, b)\rangle=-$. Moreover, $\gamma \geq \theta(a, b) \succ \beta$, contradicting $t\{\Delta, \gamma\}=\{+\}$ and establishing (iii) $\Rightarrow(\mathrm{i})$.

Lemma 1.14. If $\beta, \mu, \gamma \in \operatorname{Con} \mathbf{A}, \gamma>\mu$, and $\gamma \vee \beta>\mu \vee \beta$, then $t\{\mu, \gamma\}=\{+\}$ implies that $t\{\mu \vee \beta, \gamma \vee \beta\}=\{+\}$.

Proof. It suffices to prove the proposition when $\mu=\Delta$. By Proposition 1.13, $t\{\Delta, \gamma\}=\{+\}$ implies $\gamma \leq \mathcal{J}$, so $\gamma \vee \beta \leq \mathcal{J} \vee \beta$. But $\mathcal{J} \vee \beta / \beta$ is contained in $\mathcal{J}^{\beta}$. Applying 1.13 again, $t\{\beta, \gamma \vee \beta\}=\{+\}$.

Figure 1 demonstrates that Lemma 1.14 does not hold with "-" in place of "+". 
Definition 1.15. We define a relation on Con A. Let $\mu \approx_{+} \gamma$ if $t\{\mu, \mu \vee \gamma\}=$ $\{+\}=t\{\gamma, \mu \vee \gamma\}$.

It follows from Lemma 1.14 that $\approx_{+}$is a join congruence. We come to the main result. Recall that a join semilattice satisfies the semimodular law if $\beta \succ \alpha$ and $\gamma \geq \alpha$ imply that $\beta \vee \gamma=\gamma$ or $\beta \vee \gamma \succ \gamma$. Note that $\operatorname{Con} \mathbf{A} / \approx_{+}$is a join semilattice.

Theorem 1.16. For any algebra $\mathbf{A}$, Con $\mathbf{A} / \approx_{+}$satisfies the semimodular law.

The proof of Theorem 1.16 will depend on a technical (Proposition 1.17) proposition which is important in its own right. Proposition 1.17 is used to prove a difficult result of P. Agliano and K. Kearnes concerning semimodularity of a certain class of congruence lattices (Corollary 1.18). The proposition and corollary are presented, followed by a proof of Theorem 1.16. If $\theta>\Gamma$ in Con $\mathbf{A}, J(\theta / \Gamma)$ is computed in $\mathbf{A} / \Gamma$.

Proposition 1.17. Let $\alpha, \beta, \gamma \in$ Con $\mathbf{A}$ with $\beta>\alpha$ and $\gamma \wedge \beta=\alpha$. Suppose there exists $\alpha_{0}, \alpha_{1} \in$ Con $\mathbf{A}$ such that $\beta \geq \alpha_{1} \succ \alpha_{0} \geq \alpha, t\left\langle\alpha_{0}, \alpha_{1}\right\rangle=-$, and $t\left\{\alpha_{1}, \beta\right\}=\{+\}=t\left\{\alpha, \alpha_{0}\right\}$. If $\beta_{0}, \beta_{1}, \beta_{2}, \beta_{3} \in$ Con $\mathbf{A}$ are such that $\gamma \vee \beta \geq \beta_{3} \succ$ $\beta_{2} \geq \beta_{1} \succ \beta_{0} \geq \gamma$ and $t\left\langle\beta_{0}, \beta_{1}\right\rangle=-$, then $t\left\langle\beta_{2}, \beta_{3}\right\rangle=+$.

Proof. We may assume $\alpha=\Delta$.

Claim 1. Suppose $\beta, \alpha_{0}, \alpha_{1} \in$ Con $\mathbf{A}$ and $\beta \geq \alpha_{1} \succ \alpha_{0} \geq \Delta, t\left\{\alpha_{1}, \beta\right\}=\{+\}$, $t\left\langle\alpha_{0}, \beta_{0}\right\rangle=-$ and $t\left\{\Delta, \alpha_{0}\right\}=\{+\}$. Then $\left|J^{*}(\beta)\right|=2$.

For contradiction, suppose $\left|J^{*}(\beta)\right| \geq 3$. Then there exist $(s, t),(u, v) \in \beta-\mathcal{J}$ such that $\left|\left\{J_{s}, J_{t}, J_{u}, J_{v}\right\}\right| \geq 3$. Since $t\left\{\Delta, \alpha_{0}\right\}=\{+\}$, by Proposition 1.13 we have that $\alpha_{0} \leq \mathcal{K} \leq \mathcal{J}$. It follows that in $\mathbf{A} / \alpha_{0}$ we have $(\bar{s}, \bar{t}),(\bar{u}, \bar{v}) \in \beta / \alpha_{0}-\mathcal{J}^{\alpha_{0}}$ and that $\left|\left\{J_{\bar{s}}^{\alpha_{0}}, J_{\bar{t}}^{\alpha_{0}}, J_{\bar{u}}^{\alpha_{0}}, J_{\bar{v}}^{\alpha_{0}}\right\}\right| \geq 3$. So, it will suffice to derive a contradiction when $\alpha_{0}=\Delta$. Now, $\alpha_{1} \succ \alpha_{0}=\Delta$ and $t\left\langle\Delta_{0}, \alpha_{1}\right\rangle=-$ imply that $\left|J\left(\alpha_{1}\right)\right|=2=\left|J^{*}\left(\alpha_{1}\right)\right|$. We may suppose without loss of generality that $\left\{J_{s}, J_{t}\right\} \neq J^{*}\left(\alpha_{1}\right)$. It follows that in $\mathbf{A} / \alpha_{1},(\bar{s}, \bar{t}) \in \beta / \alpha_{1}-\mathcal{J}^{\alpha_{1}}$. But then $t\left\{\alpha_{1}, \beta\right\} \neq\{+\}$, a contradiction. This completes the proof of the claim.

Suppose $\alpha, \beta, \gamma, \alpha_{0}, \alpha_{1}, \beta_{0}, \beta_{1}, \beta_{2}$, and $\beta_{3}$ satisfy the hypotheses of the proposition. Let $(a, b) \in \beta-\mathcal{J}$. From the claim above, $J^{*}(\beta)=\left\{J_{a}, J_{b}\right\}$. We have $\gamma \vee \underline{\beta} \geq \beta_{3} \succ \beta_{2} \geq \beta_{1} \succ \beta_{0} \geq \gamma$ and $t\left\langle\beta_{0}, \beta_{1}\right\rangle=-$. Since $t\left\langle\beta_{0}, \beta_{1}\right\rangle=-$, there exists $(\bar{c}, \bar{d}) \in \beta_{1} / \beta_{0}-\mathcal{J}^{\beta_{0}}$ (so $\left.(c, d) \in \beta_{1}-\beta_{0}-\mathcal{J}\right)$. Let $c=c_{1} \cdots c_{i} c_{i+1} \cdots c_{n}=d$ witness the inclusion of $(c, d)$ in $\beta \vee \beta_{0}(=\beta \vee \gamma)$. So, $\left(c_{i}, c_{i+1}\right) \in \beta \cup \beta_{0}$, $i=1, \ldots, n-1$. If $\left(c_{1}, c_{2}\right) \in \beta_{0}$, then $\left(c_{2}, c_{n}\right) \in \beta_{1}-\beta_{0}$, moreover, we have that $\left(\bar{c}_{2}, \bar{c}_{n}\right) \in \beta_{1} / \beta_{0}-\mathcal{J}^{\beta_{0}}$ (if $\left(\bar{c}_{2}, \bar{c}_{n}\right) \in \mathcal{J}^{\beta_{0}}$, then $\left(\bar{c}_{1}, \bar{c}_{n}\right)=(\bar{c}, \bar{d}) \in \mathcal{J}^{\beta_{0}}$, a contradiction). Using the same argument with $n$ replacing 1 , we might just as well assume $\left(c, c_{2}\right),\left(c_{n-1}, d\right) \in \beta$. Using the facts that $(c, d) \notin \mathcal{J}$ and $J(\beta)=\left\{J_{a}, J_{b}\right\}$, it follows that $\left\{J_{c}, J_{d}\right\}=\left\{J_{a}, J_{b}\right\}$. That is, $t\left\langle\beta_{0}, \beta_{1}\right\rangle=-$ implies that there exists a pair $(c, d) \in \beta_{1}-\beta_{0}$ with $\left\{J_{c}, J_{d}\right\}=\left\{J_{a}, J_{b}\right\}$ and $(\bar{c}, \bar{d}) \notin \mathcal{J}^{\beta_{0}}$.

Assume for contradiction that $t\left\langle\beta_{2}, \beta_{3}\right\rangle=-$. Applying the last sentence of the previous paragraph with $\beta_{2}, \beta_{3}$ in place of $\beta_{0}, \beta_{1}$, we have that there exist $(u, v) \in$ $\beta_{3}-\beta_{2},\left\{J_{u}, J_{v}\right\}=\left\{J_{a}, J_{b}\right\}$, and $(\bar{u}, \bar{v}) \notin \mathcal{J}^{\beta_{2}}$. Let $(c, d)$ be as in the previous paragraph. Assume without loss of generality that $(u, c),(v, d) \in \mathcal{J}$. Since $(c, d) \in$ $\beta_{1} \leq \beta_{2}$, it follows that $(\bar{u}, \bar{v}) \in \mathcal{J}^{\beta_{2}}$ (even that $(\bar{u}, \bar{v}) \in \mathcal{J}^{\beta_{1}}$ with $\bar{u}, \bar{v}$ interpreted in $\left.\mathbf{A} / \beta_{1}\right)$, contradicting that $(\bar{u}, \bar{v}) \notin \mathcal{J}^{\beta_{2}}$ and establishing the proposition.

In $[\mathrm{A}-\mathrm{K}]$, the authors describe a generalization of the semigroup relation $\mathcal{J}$ which is similar to the one here. It is described for algebras in a particular variety (rather 
than "algebra by algebra" as it is done here). The interested reader can verify under the weak assumption that in a variety $V$ if $f_{i}\left(x_{1}, \ldots, x_{n_{i}}\right) \approx g\left(x_{1}, \ldots, x_{s}\right)$ implies $\left\{x_{1}, \ldots, x_{s}\right\}=\left\{x_{i_{1}}, \ldots, x_{n_{i}}\right\}$ (where $f_{i}$ is a fundamental operation and $g$ is a term), then the $[\mathrm{A}-\mathrm{K}]$ generalization and the one here are the same for $V$. (The above assumption states that there are no global "inessential" variables in a fundamental operation.) In a different notation, the authors prove

Corollary $1.18([\mathrm{~A}-\mathrm{K}])$. If $t\{\mathbf{A}\}=\{-\}$, then Con $\mathbf{A}$ satisfies the semimodular law.

Proof. Suppose $\beta \succ \alpha$ and $\gamma \wedge \beta=\alpha$. Since $t\{\mathbf{A}\}=\{-\}$, it follows by Proposition 1.17 that $\beta \vee \gamma \succ \gamma$ or $\beta \vee \gamma=\gamma$.

Proof of Theorem 1.16. For $\alpha \in$ Con $\mathbf{A}$ denote the $\approx_{+}$class of $\alpha$ by $\bar{\alpha}$. Suppose $\bar{\alpha}, \bar{\beta}, \bar{\gamma} \in \operatorname{Con} \mathbf{A} / \approx_{+}$are such that $\bar{\beta} \succ \bar{\alpha}$ and $\bar{\gamma} \wedge \bar{\beta}=\bar{\alpha}$. We will show that $\bar{\beta} \vee \bar{\gamma} \succ \bar{\gamma}$

Since $\approx_{+}$is a join congruence, we may assume that $\beta>\alpha$ and $\gamma>\alpha$ in Con $\mathbf{A}$. From $\bar{\beta} \succ \bar{\alpha}$ in Con $\mathbf{A} / \approx_{+}$, it follows that there exist $\alpha_{0}, \alpha_{1} \in$ Con $\mathbf{A}$ such that $\beta \geq \alpha_{1} \succ \alpha_{0} \geq \alpha, t\left\langle\alpha_{0}, \alpha_{1}\right\rangle=-$, and $t\left\{\alpha, \alpha_{0}\right\}=\{+\}=t\left\{\alpha_{1}, \beta\right\}$.

For contradiction, suppose there exists $\bar{\Gamma} \in \operatorname{Con} \mathbf{A} / \approx_{+}$such that $\overline{\gamma \vee \beta}>\bar{\Gamma}>\bar{\gamma}$. This is the case only if $t\{\gamma, \gamma \vee \Gamma\} \neq\{+\}$ and $t\{\gamma \vee \Gamma, \gamma \vee \Gamma \vee \beta\} \neq\{+\}$. In particular, there exist $\beta_{0}, \beta_{1}, \beta_{2}, \beta_{3} \in$ Con $\mathbf{A}$ such that $\gamma \vee \Gamma \vee \beta \geq \beta_{3} \succ \beta_{2} \geq \gamma \vee \Gamma \geq \beta_{1} \succ$ $\beta_{0} \geq \gamma(*)$, where $t\left\{\beta_{0}, \beta_{1}\right\}=-=t\left\{\beta_{2}, \beta_{3}\right\}$. Notice that $\overline{\gamma \vee \beta}>\bar{\Gamma}$ implies that $t\{\beta \vee \gamma, \beta \vee \gamma \vee \Gamma\}=\{+\}$. Applying Proposition 1.17 to $\beta \geq \alpha_{1} \succ \alpha_{0} \geq \alpha$, we are able to conclude that for any $\gamma_{0}, \gamma_{1}, \gamma_{2}, \gamma_{3}$ in Con A satisfying $\beta \vee \gamma \geq \gamma_{3} \succ \gamma_{2} \geq$ $\gamma_{1} \succ \gamma_{0} \geq \gamma$, if $t\left\langle\gamma_{0}, \gamma_{1}\right\rangle=-$, then $t\left\langle\gamma_{2}, \gamma_{3}\right\rangle=+$. Because $t\{\gamma \vee \Gamma, \beta \vee \gamma \vee \Gamma\} \neq\{+\}$ and $\approx_{+}$is a join congruence, it follows that $t\{\gamma, \beta \vee \gamma\} \neq\{+\}$. So, there exist $\theta_{0}, \theta_{1} \in$ Con $\mathbf{A}$ such that $\beta \vee \gamma \geq \theta_{1} \succ \theta_{0} \geq \gamma$ and $t\left\langle\theta_{0}, \theta_{1}\right\rangle=-$. Putting the last three sentences together, it follows that $t\left\{\gamma, \theta_{0}\right\}=\{+\}=t\left\{\theta_{1}, \beta \vee \gamma\right\}$. Also, $t\{\beta \vee \gamma, \beta \vee \gamma \vee \Gamma\}=\{+\}$, so we have $t\left\{\gamma, \theta_{0}\right\}=\{+\}=t\left\{\theta_{1}, \beta \vee \gamma \vee \Gamma\right\}$ and $t\left\langle\theta_{0}, \theta_{1}\right\rangle=-$. By Proposition 1.17, $t\left\langle\beta_{0}, \beta_{1}\right\rangle=-$ implies $t\left\langle\beta_{1}, \beta_{2}\right\rangle=+$, a contradiction. We conclude that no such $\bar{\Gamma}$ exists, completing the proof of the theorem.

Lemma 1.19. $\approx_{+}$is a complete join congruence.

Proof of Lemma 1.19. For $\alpha \in$ Con $\mathbf{A}$ we again denote the $\approx_{+}$class of $\alpha$ by $\bar{\alpha}$. The lemma is a straightforward consequence of the following claim: $\bar{\alpha}$ has a largest element which we will denote by $\alpha^{*}$. It suffices to prove the claim with $\alpha=\Delta$. Consider $\bigvee_{\Gamma \approx_{+} \Delta} \Gamma=\Delta^{*}$. By Proposition $1.13, \Gamma \approx_{+} \Delta$ if and only if $\Gamma \leq \mathcal{J}$. Since $\mathcal{J}$ is an equivalence relation, $\Delta^{*} \leq \mathcal{J}$, so $\Delta \approx_{+} \Delta^{*}$ and the claim is proved.

We complete this section with another corollary of Theorem 1.16. It places on the signed congruence lattice a strong constraint of a combinatorial flavor. If $\gamma>\mu$ in a semilattice, then $\gamma=\mu_{k} \succ \cdots \mu_{i} \succ \mu_{i-1} \cdots \succ \mu_{0}=\mu$ is said to be a saturated chain (of length $k$ ) from $\mu$ to $\gamma$. A semilattice is said to satisfy the Jordan Chain condition (or, be graded) if any two saturated chains between comparable elements have the same length. It is well known that semimodular semilattices satisfy the Jordan Chain condition.

Corollary 1.20. If $\gamma>\mu$ in Con $\mathbf{A}$, then for any two saturated chains from $\mu$ to $\gamma$ the same number of -'s appear in the signed labelling of each. 
Proof. It is not hard to see that the number of -'s occurring in a saturated path labelling is also the length of its image in Con $\mathbf{A} / \approx_{+}$, a semimodular semilattice.

Remark 1.21. E. Kiss (unpublished) produced a splitting of H-M type 1. One can label Con $\mathbf{A}$ with labels 0 and 1 by considering $\left\langle A, \mathrm{Pol}_{1} \mathbf{A}\right\rangle$. That labelling and the signed labelling are not the same. For the four-element semigroup $\mathbf{A}$ which is the product of a left-0 semigroup and a semilattice, each covering pair is labelled 0 (since $\mathrm{Pol}_{1} \mathbf{A}$ is group-free) while both - and + appear in its signed labelling.

\section{ApPliCAtions to CONGRUENCE LATtices of REGUlar SEMigroups}

Generally $\approx_{+}$is not a lattice congruence. For example, in the algebra $\mathbf{D}=$ $\langle\{0,1,2,3\}, f\rangle$ where $f(0)=1, f(1)=0, f(2)=3, f(3)=2, \approx_{+}$is not meetpreserving. There are counterexamples that involve finite semigroups. Let $S=$ $\{0, a, b, c, d, g, 1\}$ where $\{0, a, b, c, d\}$ is the five-element 0 -semigroup, $g^{2}=1, g a=$ $b=a g, g c=d=c g$. With $\theta_{1}=a c|b d| 0|g| 1, \theta_{2}=a d|b c| 0|g| 1, \theta_{i} \succ \Delta, \theta_{1} \vee \theta_{2} \succ \theta_{i}$, $t\left\langle\Delta, \theta_{i}\right\rangle=-, t\left\langle\theta_{i}, \theta_{1} \vee \theta_{2}\right\rangle=+, i=1$ and 2 . So it is somewhat surprising that $\approx_{+}$is a lattice congruence on congruence lattices of a class of regular semigroups which includes all finite regular semigroups. Such congruence lattices admit, via Theorem 1.16, a natural homomorphism onto the class of semimodular lattices. These assertions are proved below. From this point some background in semigroup theory will be assumed. F. Pastijn and M. Petrich $[\mathrm{P}-\mathrm{P}]$ constructed congruences on congruence lattices of arbitrary regular semigroups. Central among these is the socalled $U$ congruence: $\mu U \gamma$ if $\gamma \vee \mu$ is completely simple over $\gamma \wedge \mu$. For a semigroup $\mathbf{S}$, let $\mathbf{H}(\mathbf{S})$ denote the set of its homomorphic images. The exercise below (which will not be difficult for those familiar with congruence theory of regular semigroups) shows that if $\mathbf{S}$ is regular and $\mathcal{J}=\mathcal{D}$ in $\mathbf{H}(\mathbf{S})$, then the $U$ congruences can be described entirely in terms of the signed labelling.

Exercise 2.1. Suppose $\mathbf{S}$ is regular, $\mathcal{J}=\mathcal{D}$ in $\mathbf{H}(\mathbf{S})$, and $\gamma>\mu$ in Con $\mathbf{S}$.

(i) $t\{\mu, \gamma\}=\{+\}$ if and only if $\gamma$ is completely simple over $\mu$.

(ii) $t\{\mu, \gamma\} \neq\{+\}$ if and only if there exists $(a, b) \in \gamma-\mu$ such that $\{a, b\}$ is isomorphic to the two element semilattice.

Lemma 2.2. If $\mathbf{S}$ is regular and $\mathcal{J}=\mathcal{D}$ in $\mathbf{H}(\mathbf{S})$, then $\approx_{+}$is a lattice congruence.

Proof. We show that $\approx_{+}$is a meet congruence. Suppose $\Gamma>\theta, \Gamma>\beta$, and $\beta \neq \beta \wedge \theta$ in Con $\mathbf{S}$ and $t\{\theta, \Gamma\}=\{+\}$. We claim that $t\{\beta \wedge \theta, \beta\}=\{+\}$. We may assume $\beta \wedge \theta=\Delta$. Suppose for contradiction that $t\{\Delta, \beta\} \neq\{+\}$. So there exists $(a, b) \in \beta-\Delta$ such that $\{a, b\}$ is isomorphic to the two-element semilattice. Consider $\{\bar{a}, \bar{b}\}$ in $\mathbf{S} / \theta$. Since $\beta \wedge \theta=\Delta,(a, b) \notin \theta$, it follows that $(\bar{a}, \bar{b}) \in \Gamma / \theta-\Delta_{\mathbf{S} / \theta}$ and that $\{\bar{a}, \bar{b}\}$ is isomorphic to the two-element semilattice. This contradicts the assumption that $t\{\theta, \Gamma\}=\{+\}$. It is now routine to verify that $\approx_{+}$is a meet congruence.

Theorem 2.3. If $\mathbf{S}$ is a regular and $\mathcal{J}=\mathcal{D}$ in $\mathbf{H}(\mathbf{S})$, then $\operatorname{Con} \mathbf{S} / U\left(=\operatorname{Con} \mathbf{S} / \approx_{+}\right)$ satisfies the semimodular law.

\section{ACKNOWLEDGEMENT}

The author would like to thank the referee for a careful reading of the original submission. Numerous changes were suggested and followed by the author. 
P. R. Jones brought the connection between $\approx_{+}$and the $U$ congruence to the attention of the author.

\section{REFERENCES}

[A-K] P. Agliano and K. Kearnes, Congruence semimodular varieties. I, II, Algebra Universalis, 32 (1994), 224-269, 270-296. MR 95i:08010; MR 95i: 08011

[Bu-Sa] S. Burris and H. P. Sankappanavar, A course in universal algebra, Graduate Texts in Math., Springer-Verlag, Berlin and New York, 1981. MR 83k:08001

[De-D-K] J. Demel, M. Demlová, and V. Koubek, Fast algorithms for constructing minimal subalgebras, congruences, and ideals in a finite algebra, Theoret. Comput. Sci. 36 (1985) 203-216. MR 87c:68036

[D-K] M. Demlová and V. Koubek, Minimal congruence and coextensions in semigroups, Lecture Notes in Math., vol. 1320 (H. Jürgensen, G. Lallement, and H. J. Weinert, eds.), Springer-Verlag, Berlin and New York, 1988, pp. 28-84. MR 89i:20091

[H-M] D. Hobby and R. McKenzie, The structure of finite algebras, Contemp. Math., vol. 76, Amer. Math. Soc., Providence, RI, 1988. MR 89m:08001

[J1] P. R. Jones, On congruence lattices of regular semigroups, J. Algebra 82 (1983), 18-39. MR 85c:20055

[J2] Congruence semimodular varieties of semigroups, Lecture Notes in Math., vol. 1320 (H. Jürgensen, G. Lallement, and H. J. Weinert, eds.), Springer-Verlag, Berlin and New York 1986. MR 89h:20085

[P-P] F. Pastijn and M. Petrich, The congruence lattice of a regular semigroup, J. Pure Appl. Algebra 53 (1988), 93-123. MR 89j:20067

[R] J. Rhodes, A homomorphism theorem for finite semigroups, Math. System Theory 1 (1967), 289-304. MR 36:6521

[S] S. Seif, Congruence semimodular and congruence Jordan chain condition algebras characterized (submitted).

Department of Mathematics, University of Louisville, Louisville, Kentucky 40292

E-mail address: swseif01@homer.louisville.edu 